\title{
Método mejorado de simulación multiescala del viento sobre terreno complejo para la evaluación del recurso eólico
}

\author{
Enhanced method for multi-scale wind simulations over complex \\ terrain for wind resource assessment
}

\author{
A. Flores-Maradiaga ${ }^{1 *}$ \\ Recibido 11 de enero de 2016, aceptado 16 de mayo de 2016 \\ Received: January 11, 2016 Accepted: May 16, 2016
}

\begin{abstract}
RESUMEN
Debido a la variabilidad natural del viento y su fuerte dependencia en las condiciones atmosféricas locales, es necesario realizar evaluaciones exhaustivas del recurso eólico para determinar cuánta energía se puede generar en un sitio determinado [1]. Habitualmente se requiere utilizar modelos avanzados con capacidad de simulación multiescala de alta resolución para los proyectos eólicos onshore instalados sobre terreno complejo. Para tal propósito se adaptó y validó un método mejorado de simulación a gran escala (Large-Eddy Simulation, LES) en el modelo atmosférico no hidrostático MC2 de Environment Canada [2-3]. Dicha implementación ha sido validada modelando numéricamente el viento estratificado para casos típicos sobre terreno plano y montañoso, a partir de los cuales se ha comprobado que el nuevo método multiescala entrega muy buenos resultados al compararlos con otros modelos que cuentan con métodos computacionalmente intensivos [4-5].

Este modelo avanzado ahora cuenta con la capacidad de resolver estructuras multiescala del viento utilizando nuevos esquemas de inicialización y discretización que permiten una modelación numérica precisa y estable en presencia de topografía de gran impacto [6-7]. El modelo resultante puede ser utilizado para la evaluación del potencial eólico a nivel local, nacional y regional, asegurando la reducción del error numérico por sobreestimación del viento en sitios montañosos, un problema común de este tipo de herramientas computacionales. Dichos avances científicos en modelación numérica del viento aportan al estudio detallado de la instalación de parques eólicos en acantilados y cordilleras donde se espera una aceleración natural del viento.
\end{abstract}

Palabras clave: Simulación multiescala, viento, terreno complejo, modelo atmosférico, proyectos eólicos.

\section{ABSTRACT}

Due to the natural variability of wind and heavy reliance on local atmospheric conditions, it is necessary to conduct thorough wind resource assessments to determine how much energy can be generated at a given site [1]. Usually it requires advanced high resolution multi-scale models capable of simulating onshore wind farms installed on complex terrain. For this purpose, an enhanced numerical method was devised and adapted in the non-hydrostatic atmospheric model MC2 of Environment Canada with imbedded Large-Eddy Simulation (LES) capabilities for wind simulations over steep topography [2-3]. This implementation has been validated numerically by modeling the neutrally stratified wind for typical flat and mountainous terrain. From these tests it has been found that the new multi-scale method delivers very good results when compared with other models which use intensive computing methods [4-5].

\footnotetext{
1 Departamento de Ingeniería Mecánica. Universidad Técnica Federico Santa María. Av. España 1680. Casilla 110-V. Valparaíso, Chile.E-mail: alex.floresm@usm.cl

* Autor de correspondencia
} 
This advanced method now has the ability to solve multi-scale structures using a new wind initialization and time discretization schemes that allow more stable and accurate numerical modeling in the presence of high impact topography [6-7]. The resulting model can be used to assess the wind resource at local, national and regional scales, ensuring a significant error reduction due to overestimation of the wind in mountainous areas. These advancements in wind modeling provide better tools for detailed studies required for planning and installing wind farms on cliffs and ridges, where a natural acceleration of the wind is expected.

Keywords: Multiscale simulation, wind, complex terrain, atmospheric model, wind farms.

\section{INTRODUCCIÓN}

La dinámica de la Capa Límite Atmosférica (CLA) se rige por fenómenos físicos complejos cuya escala de influencia se extiende desde pocos metros hasta varios kilómetros. Esto requiere un mayor esfuerzo en el desarrollo de métodos computacionales de predicción numéricamente estables y más precisos. Particularmente, el flujo del viento sobre terreno complejo genera interacciones no lineales entre la CLA turbulenta y la superficie de la Tierra que agregan complejidad a la solución de los sistemas de ecuaciones y revelan ciertas limitaciones de los modelos numéricos convencionales. Los errores en los patrones del viento resultantes de estas simulaciones multiescala afectan las evaluaciones del recurso eólico y la planificación para los parques eólicos.

La comparación entre los resultados de las simulaciones computacionales y los experimentos realizados en túneles de viento y en terreno han demostrado que la predicción de la estructura promedio del viento sobre la topografía terrestre es independiente del modelo empleado y se logra con cierto éxito [8-13]. Sin embargo, dichas comparaciones también muestran que los resultados de los modelos de turbulencia se alteran en gran medida por las diferentes características del método seleccionado, así como por la anisotropía física de las estructuras del viento cerca de la superficie. Sobre todo se destacan los errores numéricos que surgen de los fuertes desequilibrios hidrostáticos debido a las deformaciones de la malla computacional en torno a las pendientes del terreno escarpado. La modelación precisa de la separación y recirculación de la CLA entre las montañas, acantilados y valles es actualmente uno de los principales retos en la investigación de la turbulencia atmosférica [14-15].
Cuando el viento se modela en presencia de topografía compleja es necesario seleccionar cuidadosamente los métodos numéricos para reproducir la separación del flujo, originada por los fuertes gradientes de velocidad y temperatura, así como la turbulencia cerca de la superficie. Estos fenómenos físicos a su vez interactúan con los errores computacionales de cálculo que degradan la precisión y causan inestabilidad numérica en el modelo [6, 16]. En [17] se revisaron las recientes mejoras implementadas en diversos modelos numéricos del viento y notaron que algunos de ellos generan errores significativos al simular el viento sobre terrenos con pendientes mayores de $0,2\left(\partial=11,3^{\circ}\right)$. Por tanto, se justifica el uso de modelos de turbulencia más sofisticados y correctamente validados, siendo un factor preponderante en el contexto de terreno complejo.

Coincidentemente, con el fin de aprovechar y extraer la máxima cantidad de energía del viento, los parques eólicos suelen ser instalados a la orilla de acantilados escarpados o en la cima de cadenas montañosas. En esos sitios la intermitencia e intensidad turbulenta del viento es mayor debido a la separación de la CLA lo que, a su vez, aumenta los esfuerzos de corte y la mezcla turbulenta en la capa de superficie. En esas condiciones, las alturas típicas de los aerogeneradores de envergadura encajan en la capa viscosa de superficie (10 a 20\% de la CLA) y se ven afectados por los esfuerzos cortantes dominantes. La correcta ubicación de los aerogeneradores requiere entonces un método de predicción del viento más preciso, enmarcándose en los estándares industriales para el diseño de componentes mecánicos y estructurales que resistan la fatiga del material debido a las cargas dinámicas del viento turbulento [13].

Este es un tema de gran interés para la ingeniería de sistemas eólicos, ya que los modelos numéricos 
deben ser capaces de resolver la turbulencia de microescala cerca de la superficie sin necesidad de mallas excesivamente refinadas en los sitios de interés. Por tal motivo, en las últimas décadas las técnicas de modelación numérica como la simulación "Reynolds-Averaged Navier-Stokes" (RANS) y la simulación de grandes escalas "Large-Eddy" (LES) han sentado las bases para el modelado detallado del viento. Sin embargo, estas técnicas están limitadas por los supuestos de equilibrio local y las actuales capacidades de cómputo en paralelo [17-18], lo que restringe su aprovechamiento en modelos atmosféricos de mesoescala y microescala para reproducir las circulaciones estratificadas sobre pendientes topográficas pronunciadas sin generar inestabilidad numérica [3, 14-15, 19-22].

Las evidentes ventajas y alta fidelidad de la simulación multiescala han llevado este método a convertirse en una de las tendencias dominantes del modelado del viento, con novedades interesantes como la simulación de la dinámica aeroestructural de turbinas eólicas [23]. Aunque los modelos multiescala permiten una mayor flexibilidad en la elección de los esquemas numéricos y se amplía la gama de posibles escalas a resolver, se han reportado errores numéricos del orden de $20 \%$ a $30 \%$, debido a las limitaciones en los algorítmicos, las simplificaciones cerca de la superficie, el suavizado de la malla y la asimilación de datos para simulaciones de alta resolución [20-21, 24-26]. Visiblemente, el mayor problema para el éxito de los métodos multiescala son los errores en la capa de superficie sobre fuertes pendientes y la inestabilidad numérica asociada, que requiere algoritmos numéricamente robustos capaces de resolver fenómenos transientes en presencia de terreno de alto impacto.

Por lo tanto, el objetivo de este estudio es describir y validar las posibles mejoras que se pueden implementar en modelos atmosféricos multiescala, que aplican una discretización semiimplícita a las ecuaciones de Euler para modelar el flujo compresible y no hidrostático del viento sobre orografía con pendientes pronunciadas. Para probar estas mejoras se analiza la adaptación del método LES para terreno complejo en el modelo atmosférico mesoescala MC2, incluyendo el esquema clásico de Smagorinsky para escalas de subfiltro (SFS) basado en la parametrización de viscosidad turbulenta (Eddy-Vicosity). Una descripción completa de la última versión disponible de MC2 (es decir, v4.9.8) se puede encontrar en [2], y los detalles de la primera implementación y validación sobre terreno plano del modelo MC2-LES v4.9.8 son presentados por $[3,27]$. Además, este modelo se ha actualizado con el nuevo esquema semiimplícito (N-SI) de discretización y el filtro de frecuencia RobertAsselin-Williams (RAW) descrito por [6, 28]. En lo sucesivo, esta versión mejorada se conoce como el modelo MC2-LES v4.9.9.

Las ecuaciones básicas y las últimas mejoras se describen brevemente en la segunda sección, que proporciona una visión general de la adaptación de los términos métricos adicionales en el cálculo del tensor de deformaciones para conformar el algoritmo LES a las mallas sobre terreno complejo. En la tercera sección se presentan los resultados del caso neutro sobre terreno plano para definir las constantes del esquema SFS, comprobando la sensibilidad del tamaño de malla y de los esquemas de discretización SI en el contexto del modelo MC2-LES. Asimismo, se discuten los resultados de las pruebas canónicas sobre una montaña ideal gaussiana, comparándolos con los resultados publicados en la literatura. Por último, en la quinta sección se presentan las conclusiones y se discuten ciertas recomendaciones para el trabajo futuro, proporcionando valoraciones para mejorar el método de simulación multiescala MC2-LES.

\section{ECUACIONES FUNDAMENTALES Y MEJORAS REALIZADAS EN EL MODELO MC2-LES}

El presente estudio tiene como objetivo discutir algunas mejoras recomendadas para modelos multiescala del viento, que se comprueban con la adaptación de MC2-LES v4.9.9 para simulaciones sobre terreno complejo de alto impacto. A continuación se explica cómo el sistema de ecuaciones de Euler en MC2-LES, ampliamente discutido por [3, 27] se ha modificado para obtener simulaciones más precisas y numéricamente estables en presencia de topografía escarpada.

El sistema original de ecuaciones de Euler resuelto en la versión anterior MC2-LES v4.9.8 para un régimen de flujo compresible y no hidrostático es el siguiente [2-3]: 


$$
\begin{aligned}
& \frac{d \mathbf{v}}{d t}+\left[\nabla-\gamma_{*} \mathbf{k}\right] P+\mathbf{k}\left(b-\gamma_{A} P\right)= \\
& \quad-f \mathbf{k} \times \mathbf{v}-\frac{b}{g}\left(\nabla-\beta_{A} \mathbf{k}\right) P+\mathbf{f}, \\
& \frac{d}{d t}\left(b-\gamma_{*} P\right)+N_{*}^{2} w=-b\left[\beta_{A} w+\frac{R}{c_{v}} \nabla \cdot \mathbf{v}\right]+\gamma_{A} Q, \\
& \frac{d}{d t}\left(\frac{P}{c_{*}^{2}}\right)+\nabla \cdot \mathbf{v}-\frac{g}{c_{*}^{2}} w=\frac{Q}{c_{p} T},
\end{aligned}
$$

donde $d / d t=\partial / \partial t+\mathbf{v} \cdot \nabla$ representa la derivada material, $\mathbf{v}=(u, v, w)$ la velocidad, $f$ el parámetro de Coriolis, $P=R T_{*} \ln \left(p^{\prime}\right)$ la presión generalizada, $p^{\prime}=p-p_{*}$ la perturbación de la presión, $b=g\left(T^{\prime} / T_{*}\right)$ la flotabilidad, $T^{\prime}=T-T_{*}$ la perturbación de la temperatura, $\mathbf{f}=\left(\mathrm{f}_{u}, \mathrm{f}_{v}, \mathrm{f}_{w}\right)$ las fuentes no conservativas de cantidad de movimiento y $Q$ las fuentes de calor. Las variables principales se complementan con la velocidad del sonido $c_{*}^{2}=\left(c_{p} / c_{v}\right)\left(R T_{*}\right)$ y la frecuencia de oscilación, $N_{*}^{2}=g \gamma_{*}=g\left(\beta_{A}+\gamma_{A}\right)=g^{2} / c_{p} T_{*}$ que representan los dos parámetros fundamentales del sistema, junto con las constantes $\beta_{A}=\partial \ln T_{*} / \partial z$ y $\gamma_{A}=g / c_{p} T_{*}$.

En MC2-LES v4.9.8, el sistema de ecuaciones (1) se discretiza con el esquema original semiimplícito (O-SI), que Flores-Maradiaga et al. [6] demuestra ser una formulación numéricamente inestable y ruidosa en presencia de terreno complejo. Mediante la discretización con el nuevo esquema semiimplícito (N-SI) propuesto por Flores-Maradiaga et al. [6], el sistema (1) se reformula como sigue para el modelo MC2-LES v4.9.9:

$$
\begin{aligned}
& \left.\frac{d \mathbf{v}}{d t}+\overline{(\alpha+1)(\nabla P+\mathbf{k} \hat{b}}\right)^{t}-\left(\gamma_{*}+\gamma_{A}\right) \mathbf{k} P= \\
& -f \mathbf{k} \times \mathbf{v}+\frac{\hat{b}}{g} \beta_{A} \mathbf{k} P+\mathbf{f}, \\
& \frac{d}{d t}\left[(\alpha+1) \hat{b}-\gamma_{*} P\right]+\frac{\overline{N * w}_{*}^{2}}{\alpha+1}= \\
& \quad-\left(\frac{\alpha}{\alpha+1}\right) N_{*}^{2} w+\gamma_{A} Q, \\
& \frac{d}{d t}\left(\frac{P}{c_{*}^{2}}\right)+\nabla \cdot \mathbf{v}-\frac{g}{(\alpha+1) c_{*}^{2}} w= \\
& \quad\left(\frac{\alpha}{\alpha+1}\right) \frac{g}{c_{*}^{2}} w+\frac{Q}{c_{p} T},
\end{aligned}
$$

donde las barras $\overline{[}^{t}$ denotan los términos promediados en el tiempo y resueltos implícitamente en tres niveles de tiempo (3-TL). Asimismo, $\alpha=T^{1} / T_{*}$ representa la relación de perturbación de temperatura y $\hat{b}=T^{\prime} / T$ es la nueva definición de la flotabilidad, en la cual la temperatura de referencia $\left(T_{*}\right)$ se sustituye convenientemente por la temperatura absoluta $\left(T=T^{\prime}+T_{*}\right)$. Con la aplicación del método N-SI la estructura de los términos no lineales del sistema de ecuaciones (2), resueltos explícitamente, se modifica adecuadamente para recuperar la linealidad en la relación hidrostática y asegurar la estabilidad numérica del modelo en presencia de terreno escarpado.

En términos generales, el modelo MC2-LES v4.9.9 resuelve las ecuaciones mediante la separación de las derivadas materiales de las variables principales $(d \Psi / d t)$ y los términos lineales ( $\mathrm{L}$, tratados de forma implícita) de los términos no lineales ( $\mathrm{R}$, tratados explícitamente) y los términos fuente (F). Esta separación se expresa en forma matricial como sigue:

$$
\frac{d \Psi}{d t}+\mathbf{L}=\mathbf{R}+\mathbf{F} .
$$

Luego, siguiendo lo sugerido en [3], R y F se subdividen en dos porciones, la dinámica (dedicada a las principales estructuras del flujo) y la turbulenta (dedicada a la parametrización SFS de la turbulencia), debido a que la parte volumétrica del tensor de Reynolds $\left[-2 / 3 \delta_{i j}\left(\mu_{t} S_{l l}+\bar{\rho} k\right)\right]$ se extrae de F y traslada a $\mathrm{R}$ para ser procesados junto con la presión termodinámica. Así se facilita el cálculo explícito de la parte deviatórica del tensor $\left(2 \mu_{t} S_{i j}\right)$ utilizando el algoritmo mejorado N-SI.

A saber, las porciones dinámicas $\mathrm{R}_{\mathrm{DYN}}$ y $\mathrm{F}_{\mathrm{DYN}}$ corresponden respectivamente a los términos no lineales y términos fuentes originales de sistema (1). En cambio, las porciones turbulentas $\mathrm{R}_{\mathrm{TURB}}$ y $\mathrm{F}_{\mathrm{TURB}}$ contienen respectivamente la parte volumétrica y deviatórica del tensor de Reynolds después de aplicar el filtro implícito para el método LES. Adicionalmente, se agregó un filtro de frecuencia RAW, que permite una mayor estabilidad y precisión numérica [28].

Por último, cabe resaltar que se amplía el cálculo de la difusión turbulenta para mallas que se conforman al terreno complejo, con la introducción de transformaciones del tensor métrico en la parte 
deviatórica del tensor de Reynolds incluidos entre los términos turbulentos $\left(\mathrm{F}_{\mathrm{TURB}}\right)$. Como se explica en $[2-3,29]$, dicho sistema de seguimiento del terreno se define en términos de la siguiente transformación monótona de altura para coordenadas curvilíneas y la altura del dominio $H$ [30]:

$$
\mathrm{Z}(X, Y, z)=\left[\frac{z-h(X, Y)}{H-h(X, Y)}\right] H,
$$

necesaria para reconocer las pendientes orográficas y calcular correctamente los gradientes horizontales y verticales en los puntos de la malla. Así, los términos turbulentos horizontales quedan definidos como:

$$
F_{\text {turb }}^{H}=\left\{\begin{array}{l}
F_{\text {turb-U }}^{H} \\
F_{\text {turb-V }}^{H} \\
F_{\text {turb-w }}^{H} \\
F_{\text {turb-T }}^{H} \\
0
\end{array}\right\}=\left\{\begin{array}{l}
\frac{1}{\rho}\left[\frac{\partial A_{U V}}{\partial Y_{Z}}+\frac{G_{2}}{G_{0}} \frac{\partial A_{U V}}{\partial Z}+\frac{1}{G_{0}} \frac{\partial B_{U}}{\partial Z}\right] \\
\frac{1}{\rho}\left[\frac{\partial A_{U V}}{\partial X_{Z}}+\frac{G_{1}}{G_{0}} \frac{\partial A_{U V}}{\partial Z}+\frac{1}{G_{0}} \frac{\partial B_{V}}{\partial Z}\right] \\
\frac{1}{\rho}\left[\frac{\partial A_{w}}{\partial X_{Z}}+\frac{G_{1}}{G_{0}} \frac{\partial A_{w}}{\partial Z}+\frac{\partial B_{w}}{\partial Y_{Z}}+\frac{G_{2}}{G_{0}} \frac{\partial B_{w}}{\partial Z}\right] \\
\frac{g \pi}{\rho T_{*}}\left[\frac{\partial A_{T}}{\partial X_{Z}}+\frac{G_{1}}{G_{0}} \frac{\partial A_{T}}{\partial Z}+\frac{\partial B_{T}}{\partial Y_{Z}}+\frac{G_{2}}{G_{0}} \frac{\partial B_{T}}{\partial Z}\right] \\
0
\end{array}\right\}
$$

donde $G_{0} \equiv \partial z / \partial \mathrm{Z}, \quad G_{1} \equiv \partial z / \partial X$ y $G_{2} \equiv \partial z / \partial Y$ son los gradientes jacobianos que permiten las transformaciones métricas y $\left(A_{\Psi}, B_{\Psi}\right)$ corresponden a los flujos turbulentos de cantidad de movimiento y calor. Por ejemplo, empleando la regla de la cadena para derivación, se obtienen los flujos turbulentos de cantidad de movimiento

$$
\begin{aligned}
& A_{U V}=\bar{\rho} K_{M} S\left[\frac{\partial \tilde{V}}{\partial X_{Z}}+\frac{G_{1}}{G_{0}} \frac{\partial \tilde{V}}{\partial Z}+\frac{\partial \tilde{U}}{\partial Y_{Z}}+\frac{G_{2}}{G_{0}} \frac{\partial \tilde{U}}{\partial Z}\right], \\
& B_{U}=\bar{\rho} K_{M}\left[\frac{\partial \tilde{w}}{\partial X_{Z}}+\frac{G_{1}}{G_{0}} \frac{\partial \tilde{w}}{\partial Z}\right] .
\end{aligned}
$$

Con estas mejoras se espera que el modelo multiescala MC2-LES v4.9.9 sea capaz de simular eficientemente el viento turbulento sobre terreno escarpado con una malla suficientemente refinada sin perjudicar su precisión y estabilidad numérica.

\section{VALIDACIÓN DE LAS MEJORAS PARA EL MODELO MULTIESCALA MC2-LES}

La verificación de la calidad de los resultados y los ajustes paramétricos de las simulaciones
LES, generalmente se han realizado sobre terreno plano con estratificación neutra o muy estable [5, 31-34]. Sin embargo, solo algunos investigadores han informado sobre resultados exitosos a partir de simulaciones LES sobre terreno con pendientes pronunciadas [11, 19-21]. Por tanto, nuestro reto es simular una CLA estratificada en condiciones neutras tanto sobre terreno plano como escarpado.

\section{Simulación del viento sobre terreno plano}

Con el objetivo de demostrar que este modelo multiescala cumple con las expectativas planteadas, primero se presentan los resultados del experimento clásico de la capa de Ekman sobre una superficie plana $[4-5,21]$. Esta prueba sirve para verificar si MC2-LES v4.9.9 reproduce correctamente los resultados esperados para el flujo promedio y la turbulencia de una atmósfera neutra. Igualmente, permite determinar los parámetros adecuados que aseguren simulaciones de mayor calidad con MC2LES sobre terreno montañoso, y se examina su sensibilidad para controlar la disipación, inestabilidad y precisión numérica.

Para allanar el camino de la discusión sobre las mejoras se verifica que exista una suave transición entre la versión anterior y la versión mejorada de MC2-LES (i.e., v4.9.8 y v4.9.9, respectivamente) reproduciendo la prueba canónica de la CLA neutra rotacional sobre terreno plano análoga a la capa de Ekman. Para este caso se considera que el flujo es conducido principalmente por un gradiente de presión a gran escala para una latitud de $45^{\circ}$ en el hemisferio norte, asignándole el valor clásico al parámetro de Coriolis de $f=10^{-4} \mathrm{~s}^{-1}$, que mantiene el equilibrio con un viento geostrófico de $\left(U_{G}, V_{G}\right)=(10.0,0.0) \mathrm{ms}^{-1}$. La inicialización se realiza con un perfil analítico de la capa de Ekman ligeramente perturbado con fluctuaciones aleatorias que oscilan entre $\pm 0.1 \mathrm{~ms}^{-1}$, para generar suficientes inestabilidades en el campo de flujo turbulento completamente desarrollado.

Se utiliza una malla Arakawa tipo C, con niveles escalonados verticalmente. Los tamaños geométricos de las celdas son $\Delta x=\Delta y=32 \mathrm{~m}$ y $\Delta z=4 \mathrm{~m}$, para $4.000 \mathrm{~m} \times 2.000 \mathrm{~m} \times 2.000 \mathrm{~m}$. La malla se selecciona horizontalmente periódica y uniforme en todas las direcciones. Aunque el modelo permite un espaciamiento variable en el eje vertical, se prefiere utilizar una malla estructurada y uniforme 
para no violar la condición de estabilidad lineal. Las comprobaciones preliminares indican que se requiere de una adaptación especial del tensor métrico en este tipo de algoritmos para calcular correctamente los gradientes verticales con una malla irregular no estructurada, lo que será parte de una etapa posterior de la investigación.

En los límites laterales se emplean condiciones periódicas; en la superficie se impone una condición de no deslizamiento (no-slip) con una longitud de rugosidad aerodinámica de $z_{0}=10^{-2} \mathrm{~m}$, y en la parte superior del dominio se reservan 10 niveles para una capa artificial de absorción numérica, basada en la condición de contorno de Shuman [35], para evitar la reflexión espuria de ondas acústicas.

Este conjunto de pruebas se realizó con un intervalo de tiempo de $\Delta t=4 \mathrm{~s}$ durante $300000 \mathrm{~s}$ (equivalente a $t f=30$ períodos), de tal forma que las oscilaciones inerciales debido al balance entre el gradiente de presión a gran escala y la fuerza de Coriolis llegan a un estado de equilibrio. Las estadísticas para la CLA neutra sobre terreno plano fueron promediadas en el tiempo (indicado por una barra superior) en los últimos diez períodos, y luego, promediados espacialmente (denotado con los corchetes) en cada nivel horizontal del modelo.

La sensibilidad del campo de velocidades resuelto con este modelo multiescala se mide para cada paso de tiempo con siguientes los parámetros de estacionariedad:

$$
\begin{gathered}
C_{u}=-\frac{f}{\overline{u w_{s}}} \int_{0}^{H}\left(\langle\overline{\tilde{v}}\rangle-V_{G}\right) d z \\
C_{v}=\frac{f}{\overline{v w}_{s}} \int_{0}^{H}\left(\langle\overline{\tilde{u}}\rangle-U_{G}\right) d z
\end{gathered}
$$

donde $\overline{u w_{s}}$ y $\overline{v w_{s}}$ representan los flujos totales de cantidad de movimiento de la superficie. En la Figura 1 estos dos parámetros se presentan para una simulación preliminar, que resulta igual con ambas versiones MC2-LES v4.9.8 y v4.9.9. Se logra un estado cuasiestacionario después de $t f=20$ períodos, por lo que tomamos las estadísticas únicamente para los últimos $100000 \mathrm{~s}$ en todas las simulaciones.

Con el fin de determinar los parámetros de subfiltro, este ensayo se realizó con la misma configuración
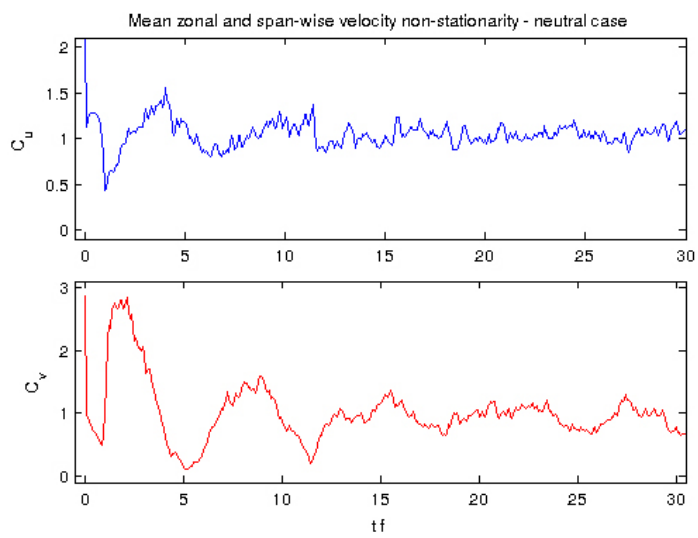

Figura 1. Parámetros de estacionariedad de la velocidad.

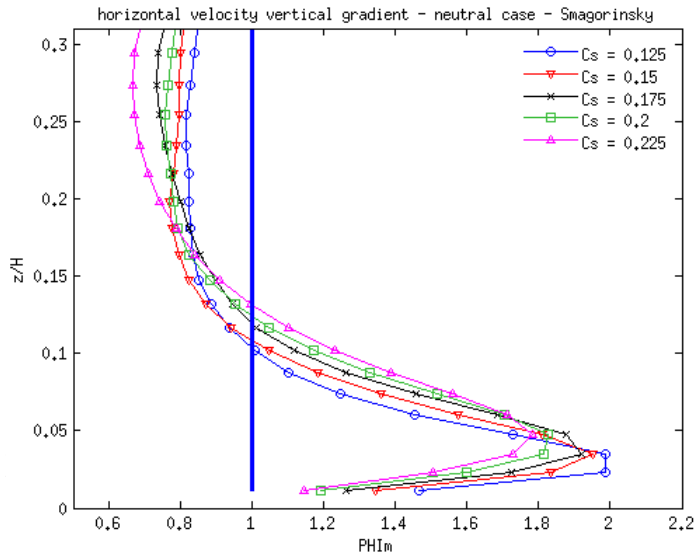

Figura 2. Gradiente vertical adimensional de la velocidad con el esquema Smagorinsky.

y condiciones de contorno utilizando dos esquemas clásicos de SFS, a saber, el Smagorinsky estándar y Deardorff. Se observaron resultados casi idénticos con ambos esquemas, por lo que sólo se discutirán los de Smagorinsky estándar. En la Figura 2 se ilustra cómo el gradiente vertical adimensional de la velocidad promedio $\Phi_{M}=\left(k z / u_{*}\right) \partial \bar{U} / \partial z$ disminuye con mayores valores de la constante paramétrica $c_{s}$.

Lo deseable es disminuir la magnitud de $\Phi_{M}$ para que se aproxime a la unidad, a pesar que valores muy altos de $c_{s}$ corresponden a una disipación numérica excesiva. Sin embargo, se debe mantener el perfil de $\Phi_{M}$ lo más próximo a la superficie $(z / H<0,1)$, lo que justifica menores valores de $c_{s}$.

Asimismo, en la Figura 3 se aprecia que la disipación de energía turbulenta es mayor cuando aumenta la 


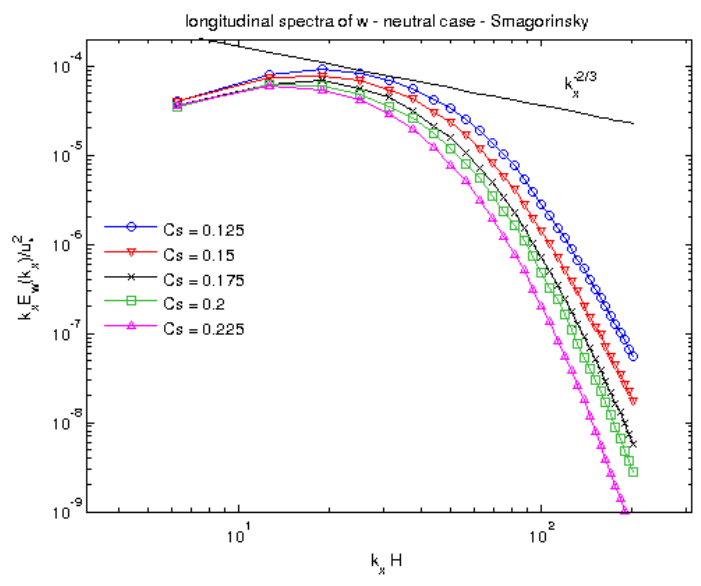

Figura 3. Espectros de energía con el esquema Smagorinsky.
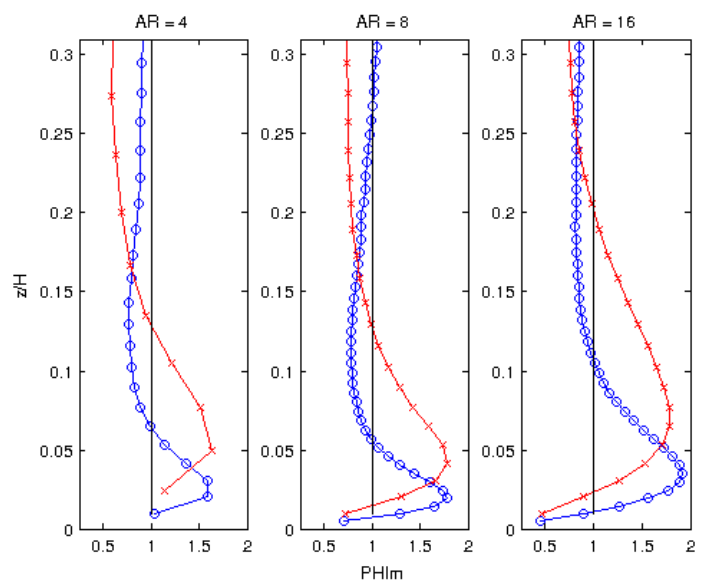

Figura 4. Proporción de resoluciones $\Delta x / \Delta z$ con tamaños $\Delta x=16 \mathrm{~m}, 32 \mathrm{~m}$ y $64 \mathrm{~m}$ versus $\Delta z=4 \mathrm{~m}$.

constante SFS. Por tanto, se definió que el valor más apropiado para las simulaciones subsiguientes es $c_{s}=0,175$.

Otro aspecto importante es el tamaño de la malla. Su efecto se examina en la Figura 4 con la proporción entre los espaciamientos horizontal y vertical $A R=\Delta x / \Delta z$. Con una proporción $A R=64 \mathrm{~m} / 4 \mathrm{~m}=16$ o mayor se aumenta el $\Phi_{M}$ y la disipación próxima a la superficie. Lamentablemente, también al refinar la malla a $A R$ $=16 \mathrm{~m} / 4 \mathrm{~m}=4$ o menos, la altura del $\Phi_{M}$ tiende a exceder la subcapa viscosa de superficie. Por ende, la mejor opción es $A R=32 \mathrm{~m} / 4 \mathrm{~m}=8$.

Este caso también se repitió empleando los dos métodos de discretización, O-SI y N-SI, para evaluar

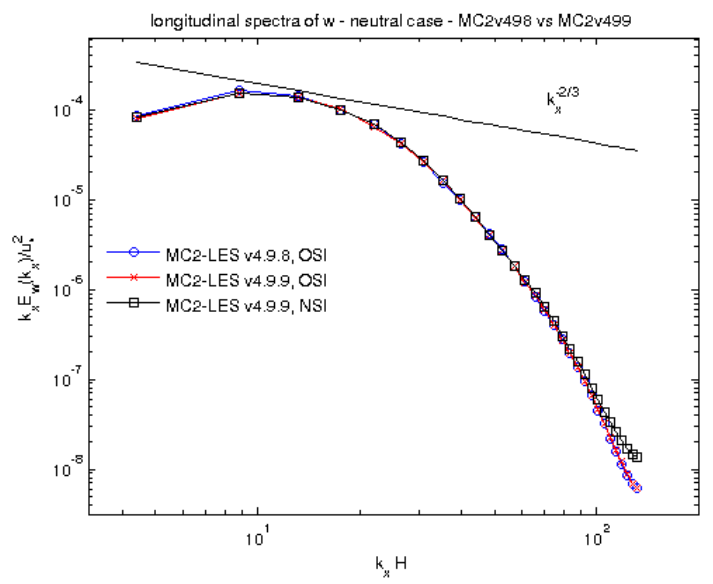

Figura 5. Espectros de energía con los esquemas O-SI y N-SI.

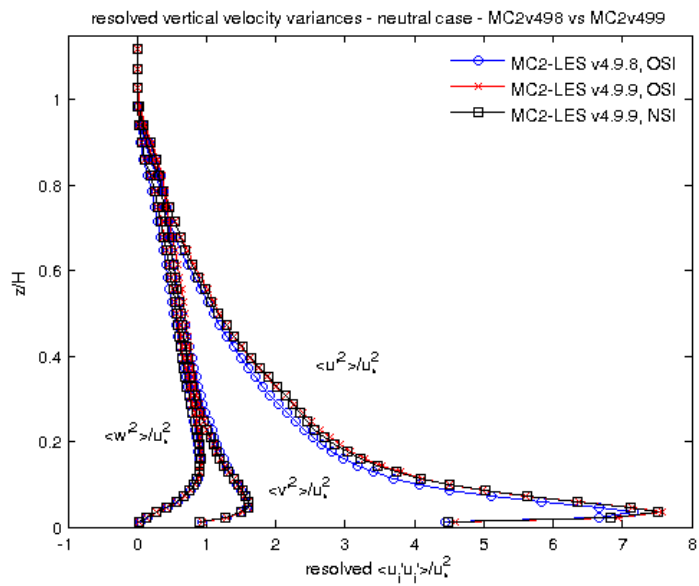

Figura 6. Varianzas de las fluctuaciones de velocidad con O-SI y N-SI.

su influencia en el modelado de la difusión turbulenta. En las Figuras 5 y 6, a partir los espectros de energía y perfiles de varianza, se observa un comportamiento muy similar entre ambos salvo que MC2-LES v4.9.9 con N-SI es menos disipativo para las estructuras de microescala cerca de la superficie.

Evidentemente, para el modo longitudinal del flujo se refleja que la energía cinética turbulenta se conserva con el modelo MC2-LES v4.9.9 y, particularmente, los perfiles esperados para el caso sobre terreno plano son independientes del tipo de discretización. Esto indica que se obtiene una mejora relativamente despreciable con una discretización más sofisticada como N-SI. A pesar de ello, se puede resaltar que con dicha implementación se reproduce eficientemente la solución clásica para la capa de Ekman. 


\section{Simulación del viento sobre una colina bidimensional}

Luego se valida el modelo con la CLA neutra sobre una colina gaussiana bidimensional, cuya altura es de $h=50 \mathrm{~m}$, anchura de base de $a=512 \mathrm{~m}$ y con una inclinación aproximada de $0,3\left(\partial \approx 16.7^{\circ}\right)$. Dicho terreno, moderadamente escarpado, puede provocar una separación y recirculación de la CLA en condiciones neutras. Este caso se inicializa con la solución de la capa de Ekman del caso anterior, logrando así un perfil dinámico más cercano al de las simulaciones sobre una colina gaussiana 2D reportados por [5, 21], que usaron el modelo multiescala WRF-LES con distintos esquemas SFS. Los resultados de Kirkil et al. [21] se etiquetan en las figuras con K12.

Para este caso se emplea el mismo tamaño de malla, intervalo de tiempo, tipo de inicialización, condiciones de contorno y parámetros SFS para modelar la turbulencia. El modelo también cuenta con la transformación métrica necesaria para conformarse al relieve y reconocer los esfuerzos resultantes del balance de cantidad de movimiento entre el viento y la superficie. Lo único que se agrega es un descentrado semilagrangiano en la discretización de los gradientes para filtrar las ondas gravitacionales y asegurar la estabilidad numérica en presencia de topografía.

A continuación se analizan fundamentalmente la aceleración del flujo en la cima de la colina y la recirculación en la ladera de sotavento. En la Figura 7 se comparan los perfiles de la velocidad promedio en tres puntos de interés, que ilustran cómo la CLA se acelera en la cima de la colina por efecto de la conservación de masa y de energía (Figura 7a). Luego se separa el flujo debido a la estratificación térmica y al cambio en el gradiente de presión local a un estado desfavorable que produce la inversión en la dirección opuesta del viento cerca de la superficie (Figura 7b). Finalmente, el flujo promedio con un gradiente de presión favorable logra restablecerse en el valle a sotavento (Figura 7c).

A partir de estas estadísticas de primer orden se comprueba que las grandes estructuras del viento sobre la colina resultan muy similares entre ambos métodos de discretización semiimplícita. Sin embargo, en la cima se observa un gradiente de

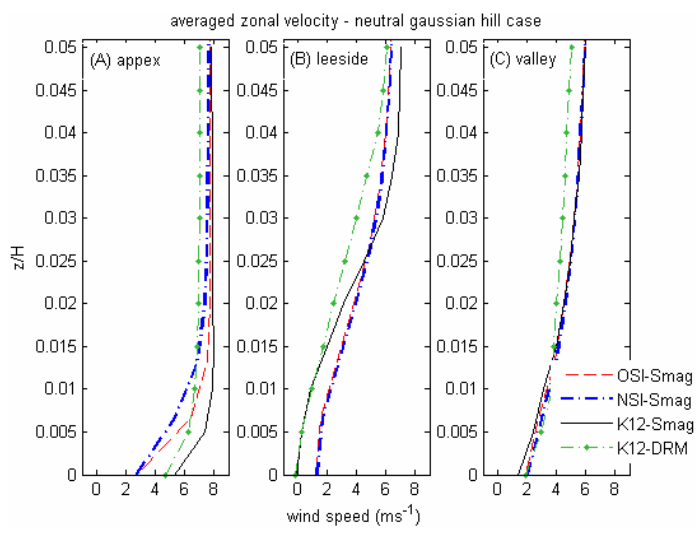

Figura 7. Velocidad promedio en (A) la cima, (B) la ladera de sotavento y (C) el valle a sotavento.

velocidad menos pronunciado con N-SI, lo que es deseable cuando se emplea la parametrización turbulenta de viscosidad turbulenta. Asimismo, los perfiles resultantes se comparan con los reportados por K12 [21], quienes utilizaron el mismo modelo SFS de Smagorinsky clásico (SMAG) y uno más sofisticado conocido como DRM para la reconstrucción espectral de las estructuras finas, filtradas explícitamente en el rango inercial de energía.

En la Figura 7a se aprecia un déficit de velocidad de superficie con respecto a los perfiles de viento K12. Posiblemente la desaceleración se deba a una excesiva disipación de energía cinética turbulenta de subfiltro. En la Figura 7b se observa una inversión menos pronunciada en la ladera de sotavento, indicando que la distancia de separación y recirculación de la CLA es menor que en las simulaciones K12. Y, en la Figura 7c se obtienen perfiles muy cercanos a K12, mostrando una rápida recuperación del régimen promedio de la CLA. Dichos resultados indican que la turbulencia atmosférica de superficie es más activa en K12, producto de la aplicación de un filtro más pequeño que refleja la energía cinética adicional de las estructuras más finas en el espectro total.

Por otra parte, para apreciar la mejora sobre terreno complejo, en la Figura 8 se muestran los flujos turbulentos del viento para la componente longitudinal en la dirección predominante del flujo. Se observa que en la cima (Figura 8a), donde el flujo 


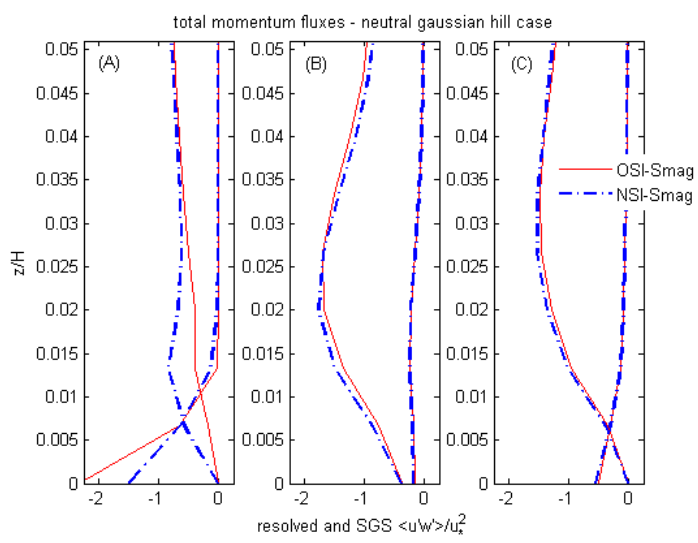

Figura 8. Flujos turbulentos verticales del viento zonal. La parte resuelta (perfil izquierdo) y la de subfiltro (perfil derecho) se muestran para la cima, ladera y valle.

se acelera, la combinación con N-SI resuelve mejor las estructuras turbulentas. El cruce entre los perfiles de las partes resueltas y de subfiltro modeladas se produce más próximo a la superficie en los resultados de N-SI. Es decir, se logra resolver más estructuras finas y no se depende tanto del modelo aproximativo SFS sobre la topografía.

Tal como se indicó en la introducción, se ha observado que el terreno complejo complica el modelado de subfiltro, por lo que es recomendable reducir su influencia en la simulación de flujos turbulentos. Por tanto, la adaptación del modelo MC2-LES v4.9.9 con $\mathrm{N}-\mathrm{SI}$ para modelación del viento sobre topografía escarpada recupera la eficiencia necesaria en estos casos.

Para visualizar las estructuras en la capa de superficie obtenido con ambas versiones, en la Figura 9 se muestran imágenes instantáneas del viento obtenidas con ambos esquemas de discretización SI a una altura de $10 \mathrm{~m}$ sobre el terreno.

Aunque similares, se reconocen ciertas diferencias cualitativas producto de las mejoras en MC2-LES. En ambos se visualiza el viento de la magnitud esperada y con una inclinación generada por el efecto rotacional de Coriolis, lo que se ajusta bien a los resultados de Kirkil et al. [21]. Sin embargo, con N-SI el flujo sobre la colina está correctamente confinado a la zona donde interactúa el viento con la pendiente topográfica; en cambio, con O-SI se expande fuera de esa zona de influencia, mostrando
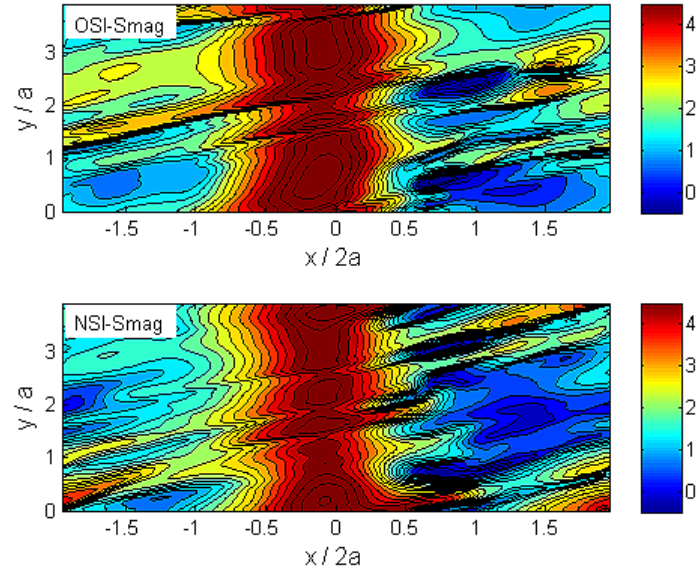

Figura 9. Viento $\left(\mathrm{ms}^{-1}\right)$ de superficie a $10 \mathrm{~m}$ sobre el suelo.

alguna aceleración espuria en la ladera de barlovento. Esto tiene relación con la sobreestimación del viento de superficie mostrada en la Figura 7a. También, es evidente que el viento forma una burbuja de separación y recirculación mejor definida en la ladera de sotavento con N-SI en relación a O-SI.

\section{CONCLUSIONES}

A partir de las estadísticas de primer y segundo orden se observa que no hay variaciones significativas entre las dos versiones de modelo, con y sin las mejoras, para simulaciones de la CLA neutra sobre terreno plano. Es decir, las mejoras numéricas no tienen un efecto notable en el modelado LES de la difusión turbulenta sobre terreno plano. Sin embargo, con estas mejoras para métodos multiescala se logra reproducir eficazmente los resultados obtenidos con el modelo MC2-LES v4.9.8, previamente validado por Gasset et al. [3]. Es decir, se comprueba que los cambios implementados en el código no perjudican su rendimiento.

Para las simulaciones sobre topografía escarpada, donde la CLA neutra se separa produciendo una zona de inversión y recirculación turbulenta en la ladera de sotavento, el método multiescala mejorado es capaz de resolver mejor las estructuras finas especialmente en la capa de superficie sin necesidad de refinar localmente la malla. Asimismo, se reduce el gradiente vertical de la velocidad promedio y se previene la sobreestimación del viento en la cima de las colinas. Aunque modestas, dichas mejoras 
reflejan un avance en la correcta definición del flujo a lo largo de una cadena montañosa permitiendo evaluar el recurso eólico con mayor precisión sobre topografía de alto impacto.

Se recomienda investigar más a fondo la adaptación del tensor métrico en el cálculo de la difusión turbulenta para mallas irregulares no estructuradas. Además, se deben examinar los esquemas de subfiltro en presencia de terreno complejo y el efecto de la estratificación térmica de la CLA.

\section{AGRADECIMIENTOS}

Los autores reconocen el valioso aporte del Dr. Nicolas Gasset en la adaptación del método LES para MC2-LES v4.9.9. También, se agradece al Dr. Claude Girard y al equipo de Recherche en Prévision Numérique (RPN), del Servicio Meteorológico de Canadá, por proporcionar el asesoramiento, el apoyo técnico y las bibliotecas de MC2 para implementar las mejoras propuestas.

Asimismo, agradecemos a la Fundación WESNet y a la Universidad Técnica Federico Santa María por la financiación respectiva de la investigación a través de los proyectos WESNet 1.1-a y USM 25-15-11.

\section{REFERENCIAS}

[1] J. Manwell, J. McGowan and A. Rogers. "Wind energy explained: theory, design and application". John Wiley \& Sons, pp. 689. London, Great Britain. 2010.

[2] C. Girard, R. Benoit and M. Desgagné. "Finescale topography and the MC2 dynamics kernel". Monthly Weather Review. Vol. 133, pp. 1463-1477. 2005.

[3] N. Gasset, R. Benoit and C. Masson. "Implementing large-eddy simulation capability in a compressible mesoscale model". Monthly Weather Review. Vol. 142, pp. 2733-2750. 2014.

[4] F.K. Chow, M. Xue and J.H. Ferziger. "Explicit filtering and reconstruction turbulence modeling for large-eddy simulation of neutral boundary layer flow". Journal of Atmospheric Sciences. Vol. 62, pp. 2058-2077. 2005.

[5] G. Kirkil, J. Mirocha, E. Bou-Zeid, F.K. Chow and B. Kosović. "Implementation and evaluation of dynamic sub-filter scale stress models for large-eddy simulation using WRF". Monthly Weather Review. Vol. 140, pp. 266-284. 2012.

[6] A. Flores-Maradiaga, R. Benoit, C. Girard, C. Masson and M. Desgagné. "On the enhancement of numerical stability and noise control for mesoscale modelling over steep complex terrain". In press in Theoretical and Computational Fluid Dynamics. Springer. 2016.

[7] A. Flores-Maradiaga, P. Pham, R. Benoit and C. Masson. "Statistical dynamical downscaling of strongly stratified surface wind over steep complex terrain with the enhanced MC2 mesoscale model". In press Wind Engineering, University of Massachusetts Press. 2016.

[8] H. Kim and V. Patel. "Test of turbulence models for wind flow over terrain with separation and recirculation". BoundaryLayer Meteorology. Vol. 94, pp. 5-21. 2000.

[9] Y. Nagano, H. Hattori and T. Irikado. "Prediction of flow over complex terrain using turbulence model". Proceedings of the TDE-Conference, JSME. 2001.

[10] F. Castro, J. Palma and A. Silva Lopes. "Simulations of the Askervein flow. Part 1: Reynolds averaged Navier-Stokes equations ( $k-\varepsilon$ turbulence model)". Boundary-Layer Meteorology. Vol. 107, pp. 501-530. 2002.

[11] A. Weigel, F.K. Chow, F.K., M. Rotach, R. Street and M. Xue. "High resolution large-eddy simulations of flow in a steep Alpine valley. Part 2: flow structure and heat budget". Journal of Applied Meteorology and Climatology. Vol. 45, pp. 87-107. 2006.

[12] A. Silva Lopes, J. Palma and F. Castro. "Simulations of the Askervein flow. Part 2: Large eddy simulations. Boundary-Layer Meteorology. Vol. 125, pp. 85-108. 2007.

[13] K. Ayotte. "Computational modeling for wind energy assessment". Journal of Wind Engineering and Industrial Aerodynamics. Vol. 96, pp. 1571-1590. 2008.

[14] T. Allen and A. Brown. "Large-eddy simulation of turbulent separated flow over rough hills". Boundary-Layer Meteorology. Vol. 102, pp. 177-198. 2002.

[15] K. Lundquist, F.K. Chow and J. Lundquist. "An immersed boundary method for the Weather 
Research and Forecast model". Monthly Weather Review. Vol. 138, pp. 796-817. 2010.

[16] P. Bénard, J. Mašek and P. Smolíková. "Stability of leapfrog constant-coefficients semi-implicit schemes for the fully elastic system of Euler equations: case with orography". Monthly Weather Review. Vol. 133, pp. 1065-1075. 2005. DOI: 10.1175/ MWR2907.1

[17] J. Sumner, C. Sibuet and C. Masson. "CFD in wind energy: The virtual, multiscale wind tunnel". Energies. Vol. 1, pp. 1-26. 2010.

[18] P. Mason and D. Thomson. "Stochastic backscatter in large eddy simulations of boundary layers". Journal of Fluid Mechanics. Vol. 242, pp. 51-78. 1992.

[19] F.K. Chow and R. Street. "Modeling unresolved motions in LES of field-scale flows". 15th Symposium on Boundary Layers and Turbulence. 2002.

[20] A. Bechmann and N. Sorensen. "Hybrid RANS/LES method for wind flow over complex terrain". Wind Energy. Vol. 13, pp. 36-50. 2010.

[21] J. Mirocha, F. Chow, J. Lundquist and K. Lundquist. "Improved subfilter turbulence modeling for large eddy simulation using WRF". 7th Symposium on the Urban Environment, American Meteorology Society. 2007.

[22] K. Lundquist, F.K. Chow and J. Lundquist. "An immersed boundary method for the Weather Research and Forecast model". Monthly Weather Review. Vol. 138, pp. 796-817. 2010.

[23] P. Fleming, M. Churchfield, A. Scholbrock, J. Michalakes, K. Johnson and P. Moriarty. "The SOWFA super-controller: a highfidelity tool for evaluating wind plant control approaches. National Renewable Energy Laboratory, NREL/CP-500057175. 2013.

[24] J.P. Pinard, R. Benoit and W. Yu. "A WEST wind climate simulation of the mountainous Yukon". Atmosphere-Ocean. Vol. 43, pp. 259282. 2005.

[25] J.P. Pinard, R. Benoit, J.D. Wilson, Mesoscale wind climate modelling in steep mountains, Atmosphere-Ocean. Vol. 47, pp. 63-78. 2009. DOI: 10.3137/AO922.2009
[26] N. Gasset, M. Laudry and Y. Gagnon. "A comparison of wind flow models for wind resource assessment in wind energy applications". Energies. Vol. 5, pp. 42884322. 2012. DOI: 10.3390/en5114288.

[27] N. Gasset. "Refinement of a mesoscale model for large-eddy simulation". PhD Thesis. École de Technologie Supérieure. Montreal, Canada. 2014.

[28] P.D. Williams. "The RAW filter: an improvement to the Robert-Asselin filter in semi-implicit integrations". Monthly Weather Review. Vol. 139, pp. 1996-2007. 2011. DOI: 10.1175/2010MWR3601.1.

[29] S.J. Thomas, C. Girard, R. Benoit, M. Desgagné and P. Pellerin. "A new adiabatic kernel for the MC2 model". AtmosphereOcean. Vol. 36, 241-270. 1998.

[30] T. Gal-Chen and R.C. Somerville. "On the use of a coordinate transformation for the solution of Navier-Stokes equations". Journal of Computational Physics. Vol. 17, pp. 209-228. 1975.

[31] B. Kosović and J. Curry. "A large-eddy simulation study of a quasi-steady, stably stratified atmospheric boundary layer". Journal of Atmospheric Science. Vol. 57, pp. 1052-1058. 2000.

[32] P. Drobinski, P. Carlotti and J. Redelsperger. "Numerical and experimental investigation of the neutral atmospheric surface layer". Journal of Atmospheric Science. Vol. 64, pp. 137-149. 2007.

[33] J. Redelsperger, F. Mahé and P. Carlotti. "A simple and general subgrid model suitable both for surface layer and free-stream turbulence". Boundary-Layer Meteorology. Vol. 101, pp. 375-408. 2001.

[34] I. Senocak, A. Ackerman, M. Kirkpatrick, D. Stevens and N. Mansour. "Study of nearsurface models in large eddy simulations of a neutrally stratified atmospheric boundary layer". (Part I). Annual Research Briefs. Center for Turbulence Research, NASA Ames/Stanford University. 2004.

[35] F. Shuman. "Numerical methods in weather prediction: (II) smoothing and filtering". Monthly Weather Review. Vol. 85, pp. 357361. 1957. 